\title{
The influence of gestational trimester, physical activity practice and weight gain on the low back and pelvic pain intensity in low risk pregnant women
}

\author{
Vanessa Patrícia Soares de Sousa*, Alethéa Cury, Laiane Santos Eufrásio, \\ Sara Estéfani Soares de Sousa, Carolina Bezerra Coe and Elizabel de Souza Ramalho Viana \\ Department of Physical Therapy, Federal University of Rio Grande do Norte, Natal, Rio Grande do Norte, RN, \\ Brazil
}

\begin{abstract}
.
BACKGROUND: Low back and pelvic pain in pregnant women is a clinical condition of which the etiology is multifactorial. Thus, various variables can influence the low back and pelvic pain's intensity.

OBJECTIVE: The purpose of this study was to analyze the influence of the gestational trimester, practice of physical activity and weight gain on the intensity of low back and pelvic pain in low risk pregnant women.

METHODS: Two hundred and sixty-seven pregnant women participated in this study. The gestational age, body mass index, weight gain, physical activity practice and the low back and pelvic pain were evaluated.

RESULTS: We found a significant difference $(P=0.02)$ in pain intensity, when comparing active and sedentary pregnant women. No significant differences were found when comparing pain intensity between the gestational trimesters $\left(2^{\text {nd }}\right.$ versus $3^{\text {rd }}$; $P=0.60)$. There was no significant relation between the weight gain and pain intensity $(r=0.03 \mid P=0.28)$. The multivariate analysis indicated that sedentary pregnant women have a higher risk $(P=0.001)$ of intense pain and the pain is not influenced by the weight gain $(P=0.08)$ and the gestational trimester $(P=0.98)$.

CONCLUSIONS: Sedentary pregnant women have $30 \%$ more chances to have higher pain intensities when compared to the
\end{abstract} active women, regardless of the gestational trimester and weight gain.

Keywords: Pregnancy, low back pain, gestational age, exercises, body weight

\section{Introduction}

Low back and pelvic pain (LBPP) are considered the main cause of incapacity in humans [1]. It is defined as a pain located between the ribs and the gluteus area which may irradiate to the lower limbs [2].

\footnotetext{
${ }^{*}$ Corresponding author: Vanessa Patrícia Soares de Sousa, Department of Physical Therapy, Federal University of Rio Grande do Norte, Rua Valença, 2826, conj. Santa Catarina. Natal, Rio Grande do Norte, Brasil. Tel.: +55 8498702 7110; E-mail: vanessafisio@ gmail.com.
}

Low back and pelvic pain in pregnant women is an important clinical condition that must be considered, since it directly affects these women's daily activities [3]. The etiology of LBPP is multifactorial and frequently linked to biomechanical, hormonal and vascular changes. In addition to these alterations, the age, economic status, previous pregnancies, body mass index (BMI), familial history of LBPP and history of hypermobility are some of the risk factors that may be associated with this pathology [4,5].

Studies suggest that LBPP is reported by more than $50 \%$ of the pregnant women and the intensity of this

ISSN 1053-8127/19/\$35.00 (C) 2019 - IOS Press and the authors. All rights reserved

This article is published online with Open Access and distributed under the terms of the Creative Commons Attribution Non-Commercial License (CC BY-NC 4.0). 
pain increases with the gestation's advance $[4,6,7]$. Despite the prevalence and severity of the symptoms, lots of pregnant women do not search treatment for LBPP, either because of the lack of knowledge about possible treatments or because they consider it a normal or unmanageable pathology [4]. On the other hand, the women who received physiotherapeutic treatment, through orientations about LBPP prevention and treatment, related a better quality of life in pregnancy [8].

Research also reveals the importance of prescribing exercises as a first-line treatment, in order to avoid the use of medication during pregnancy $[4,8]$. However, a systematic review about the effect of exercises during and after pregnancy, written by $\mathrm{Sd}$ and Pennick in 2015 [9], showed that the results from the controlled and randomized clinical trials are small and not conclusive.

The result of the research has suggested that the weight gain during pregnancy, the advance of pregnancy and practice of physical activity, may influence the LBPP intensity $[10,11]$. The lack of physical activity and weight gain are related to back pain. A sedentary lifestyle allied to a deficiency of the musculoskeletal system and a spine overload makes the population more prone to have low back and pelvic pain [12].

In this way, the study of the variables that may influence the low back and pelvic pain's intensity is an important guiding point to the clinical practice, since it has been shown that this pain may persist throughout pregnancy and, often, during the post-partum $[8,13]$. Therefore, the main aim of this study was to analyze the influence of the gestational trimester, practice of physical activity and weight gain on the intensity of low back and pelvic pain in low risk pregnant women.

\section{Methods}

This study was approved by the Ethics Committee for Research with Humans of Rio Grande do Norte Federal University (UFRN) under protocol number 719.939 e CAAE 30403414.8.0000.5537. All volunteers have signed the Free and Clarified Consent Term (FCCT).

\subsection{Participants and context}

The sampling process was non-probabilistic. The volunteers were recruited, from $16^{\text {th }}$ gestation week, to the preparatory course for pregnancy, delivery and postpartum - PCPDP, promoted monthly by the Physical Therapy Department of UFRN. We adopted the

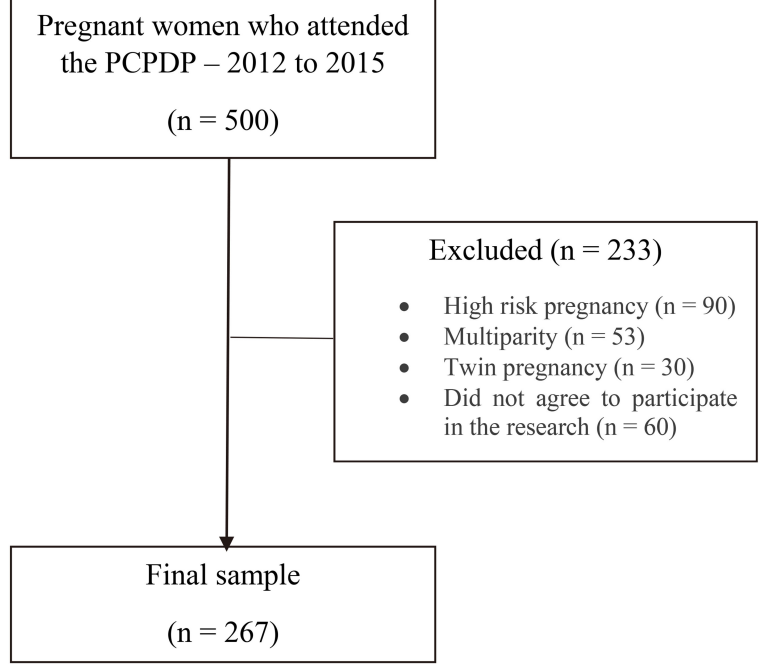

Fig. 1. Search sample flowchart.

following inclusion criteria: low-risk pregnancy, nulliparous and single fetus. The activities carried out at the PCPDP were: educational lectures on issues related to gestation, childbirth, postpartum and baby care. In addition, the practice of physical activity ranged from mild to moderate intensity.

To the sample calculation, we considered the total of pregnant women who attended the PCPDP from 2012 to 2015 ( $n=500$ ). The following data were included in the software G Power (University of Kiel, Germany, 2014): $\alpha=0.05$; $\beta$ (power) $=0.95$; effect size $=0.5$. According to this calculation the ideal $\mathrm{n}$ to our study was 218. At the end of the research we reached the number of 267 volunteers (Fig. 1).

\subsection{Evaluation tools}

The variables were collected using an evaluation form, elaborated by the responsible researchers, and divided into the following groups: sociodemographic data (age, years of education, family income, marital status), obstetric data (gestational age and trimester), anthropometric and lifestyle data (weight, height, BMI, weight gain and physical activity practice).

The weight gain calculation was made from a subtraction of the weight in the moment of evaluation and pre-gestational weight. For the pre-gestational body mass index (BMI), the weight of the woman before pregnancy was considered and classified as: underweight (BMI, $\left.18.5 \mathrm{Kg} / \mathrm{m}^{2}\right)$, normal range $(\geqslant 18.5 \mathrm{e}$ $\left.25 \mathrm{Kg} / \mathrm{m}^{2}\right)$, overweight $\left(\geqslant 25 \mathrm{e}<30 \mathrm{Kg} / \mathrm{m}^{2}\right)$, or obese $\left(\geqslant 30 \mathrm{Kg} / \mathrm{m}^{2}\right)[14]$. The nutritional state diagnose was 
Table 1

Sociodemographic, obstetric, anthropometric and lifestyle characteristics $(n=267)$

\begin{tabular}{lccc}
\multicolumn{1}{c}{ Variables } & $\begin{array}{c}\text { Second trimester } \\
(n=154)\end{array}$ & $\begin{array}{c}\text { Third trimester } \\
(n=113)\end{array}$ & $\begin{array}{c}\text { Total } \\
(n=267)\end{array}$ \\
\hline Socialdemographic data & & & \\
Age (years) & $29.83 \pm 4.74$ & $29.77 \pm 3.72$ & $29.80 \pm 4.33$ \\
Years of study & $16.24 \pm 3.22$ & $16.62 \pm 3.49$ & $16.41 \pm 3.34$ \\
Marital status & & & \\
$\quad$ With lifepartner & $94.1 \%(145)$ & $92.2 \%(105)$ & $93.6 \%(250)$ \\
$\quad$ Without lifepartner & $1.9 \%(3)$ & $0.9 \%(1)$ & $1.5 \%(4)$ \\
Obstetric data & & & \\
Gestational age (weeks) & $20.7 \pm 3.49$ & $30.05 \pm 2.31$ & $24.68 \pm 5.52$ \\
Trimesters & & & \\
$\quad$ Second & & & $57.7 \%(154)$ \\
$\quad$ Third & & & $42.3 \%(113)$ \\
Anthropometric and lifestyle data & $60.04 \pm 11.26$ & $65.47 \pm 10.65$ & $62.32 \pm 11.31$ \\
Pre-gestational weight $(\mathrm{kg})$ & $66.05 \pm 10.36$ & $71.30 \pm 10.25$ & $68.26 \pm 10.62$ \\
Gestational weight $(\mathrm{kg})$ & $1.62 \pm 0.06$ & $1.62 \pm 0.06$ & $1.62 \pm 0.06$ \\
Height (m) & $22.79 \pm 3.91$ & $24.78 \pm 3.78$ & $23.62 \pm 3.97$ \\
Pre-gestational BMI $\left(\mathrm{kg} / \mathrm{m}^{2}\right)$ & $24.92 \pm 4.09$ & $27.01 \pm 3.66$ & $25.79 \pm 4.05$ \\
Gestational BMI (kg/m $\left.{ }^{2}\right)$ & $5.97 \pm 4.20$ & $5.86 \pm 3.63$ & $5.92 \pm 3.96$ \\
Weight gain & & & \\
Physical activity & $35.7 \%(55)$ & $45.1 \%(51)$ & $39.7 \%(106)$ \\
Active & $63.6 \%(98)$ & $54 \%(61)$ & $59.6 \%(159)$ \\
Sedentary & & & \\
\hline
\end{tabular}

Note: there was loss of data for the variable.

made according to the gestational age, using the Atalah's curve (1997). We used the BMI limit values, by gestational week to the classification into: underweight, normal range, overweight and obesity [15].

Regarding the practice of physical activity, the volunteers were asked: "Do you practice physical activity (low to moderate intensity), at least twice a week, with minimum duration of 50 minutes (by episode)?" If the answer was "yes", the woman was considered active; otherwise, sedentary [16].

The low back and pelvic pain evaluation was made by asking the volunteer initially "Do you feel low back and/or pelvic pain?". If the answer was positive, the pregnant woman would indicate the intensity of the pain using the Visual Analogue Scale (VAS) to localize pain in the human figure [17].

\subsection{Statistical analysis}

To characterize the sample, we used descriptive analysis (mean, standard deviation, absolute and relative frequencies). In order to test the quantitative variables normality we used Shapiro-Wilk's test. The comparison of the pain intensity between active and sedentary pregnant women and between those in the second and third gestational trimester was tested by MannWhitney U test. Spearman's correlation test was used to identify the relation between weight gain and pain intensity.
To analyze the influence of physical activity practice (categorical variable: active versus sedentary), gestational trimester (categorical variable: second versus third) and weight gain (numeric variable) over low back and pelvic pain intensity (numeric variable), we used Poisson regression. $P$ value below 0.05 was considered significant.

\section{Results}

Sociodemographic and obstetric characterization of the participants showed that $61.8 \%(n=165)$ had an income greater than 4 salary, $71.9 \%(n=192)$ were married and $57.7 \%(n=154)$ were in the third trimester. Further information is displayed in Table 1.

The bivariate analysis showed a significant difference $(P=0.02|\mathrm{U}=5106.00| \mathrm{IC} 95 \%=0.01-0.05)$ in pain intensity, when comparing active (median $=$ $3.0 \mid$ quartile $25=0.0 \mid$ quartile $75=5.0$ ) and sedentary $($ median $=4.0 \mid$ quartile $25=1.0 \mid$ quartile $75=$ 6.0) pregnant women. However, when comparing pain intensity between the gestational trimesters, no significant differences were found $(P=0.60 \mid \mathrm{U}=6150.50$ | IC95\% $=0.56-0.68)$. There was no significant relation between weight gain and pain intensity $(r=0.03$ | $P=0.28)$.

The multivariate analysis indicated that sedentary pregnant women have $30 \%$ more chances $(P=0.001)$ 
Table 2

Multivariate analysis of the relation among physical activity practice, gestational trimester, weight gain and pain intensity

\begin{tabular}{lccccc}
\hline & $\begin{array}{c}\text { Chi-squared } \\
\text { test }\end{array}$ & $P$ & Exp (B) & \multicolumn{2}{c}{$\begin{array}{c}\text { Confidence } \\
\text { interval 95\% }\end{array}$} \\
\cline { 3 - 6 } & 12.44 & $\mathbf{0 . 0 0 1}$ & 1.30 & 1.12 & 1.50 \\
\hline Sedentary & - & - & 1 & - & - \\
Active & 1.54 & 0.21 & 0.91 & 0.79 & 1.05 \\
Second trimester & - & - & 1 & - & - \\
Third trimester & 3.06 & 0.08 & 0.98 & 0.96 & 1.00 \\
Weight gain & & & & &
\end{tabular}

to have more intense pains, when compared to the active women. Pain is not influenced by the weight gain $(P=0.08)$ and the gestational trimester $(P=0.98)$ (Table 2).

\section{Discussion}

The aim of this study was to analyze the influence of physical activity practice, gestational trimester and weight gain influence on the intensity of low back and pelvic pain. The main result suggests that sedentary pregnant women have $30 \%$ more chances to feel more severe pain, when compared to the active women $(\mathrm{OR}=1.30 ; P=0.001)$, regardless of the gestational trimester $(\mathrm{OR}=0.91 ; P=0.21)$ and weight gain $\left(r^{2}=0.98 ; P=0.08\right)$.

Physical activity during pregnancy promotes lots of benefits, such as: reducing the risk of overweight, gestational diabetes, pre-eclampsia, preterm birth, varicose veins and deep vein thrombosis [18]. These benefits also include the decrease of low back and pelvic pain intensity, as shown by the study by Da Silva et al. [17], in which they submitted 26 pregnant women to a physical activity protocol for 1 month. The results of this research showed a significant decrease in the LBPP, measured by the Visual Analog Scale (VAS), when compared the values before and after the intervention. A randomized controlled clinical trial with 45 volunteers (20 in the experimental group and 22 in the control group), also showed similar results: pregnant women submitted to a physical activity protocol, which included aerobic and muscle strengthening activities, presented lower pain intensity when compared to the control group [19]. These findings corroborate with the results of our study.

Physiologically, during pregnancy low back and pelvic muscles are gradually overloaded, due to an increase in the total body mass and uterus size [20]. These factors take the abdominal muscles to its maximum stretching, resulting in an unfavorable length ten- sion relationship to the force generation, which causes a decrease in low back and pelvic stability [21,22]. Thus, although paravertebral muscles are composed mostly by muscle fibers type I, the muscle overload and imbalance, characteristics of the biomechanical changes due to pregnancy, may result in LBPP [22]. Contrary to the pregnant body adaptations, the regular practice of physical activity results in a cardiorespiratory fitness, endurance and muscle strength improvement, and, consequently, decreases low back and pelvic pain intensity [23].

Additionally, the regular practice of physical activity is opposite to a sedentary lifestyle, which is defined as the execution of activities that spend little metabolic energy and is connected to unfavorable health conditions [24]. This concept corroborates with our findings, since sedentary women presented $30 \%$ more chances to report greater pain intensities than the active women in our study.

In this way, the professionals responsible for assisting pregnant women should encourage them to keep active during pregnancy, prescribing physical activities that respect the principles of modality, duration, frequency, intensity and progression mode [25].

We did not observe the influence of the third gestational trimester variable over LBPP intensity $(\mathrm{OR}=$ $0.91 ; P=0.21)$. A cross-sectional study [26], similar to ours, with 235 pregnant women, found that the main factors related to LBPP are: satisfaction with work, BMI, quality of life and previous LBPP. There was no association between gestational trimester and LBPP, corroborating with our findings. On the other hand, the research done by Mota et al. [4] with 105 volunteers, found that the LBPP intensity increased with the advance of pregnancy. However, it is important to consider the following differences between our study and Mota's. In the current study, the interviews were done with the pregnant women experiencing the pain during the data collection, including physical activity practice data, while in Mota's study the interviews took place during the post-partum, with questions about their pregnancy but with no reports about the quality of life during pregnancy. A possible argument for the lack of influence of the gestational trimester on LBPP refers to this lifestyle characteristic of our sample. Proportionally, third trimester pregnant women were more active $(45.1 \%)$ than women in their second trimester $(35.7 \%)$. Considering that the practice of physical activity decreases the risk of high pain intensities, this can be a possible explanation for this finding.

Lastly, we found that the weight gain did not influence the LBPP intensity $\left(r^{2}=0.98 ; P=0.08\right)$. 
This is justified by the sample anthropometric characteristics. According to the values standardized by WHO (1995) to define pre-gestational nutritional state, our sample was at the normal range (pre-gestational BMI: $\geqslant 18.5$ e $<25 \mathrm{~kg} / \mathrm{m}^{2}$ ) [14]. Other than that, the main weight gain at this study was not significant $(5.97 \pm 4.20$ e $5.86 \pm 3.63$, in second and third trimester, respectively). The Institute of Medicine, National Academy of Sciences (1990) advocates a weight gain of $1.6 \mathrm{~kg} /$ week in the first trimester and $0.4 \mathrm{~kg} /$ week in the second and third trimesters. The weight gain in this study was 4.4 to $5.6 \mathrm{~kg}$ and 8.4 to $9.2 \mathrm{~kg}$ (second and third trimesters, respectively). Therefore, the weight gain at this study did not surpass the normality range, and this fact can justify the noninfluence of the weight gain on the LBPP intensity. In 2016, Bliddal et al. [27] conducted a study to identify the factors associated with musculoskeletal disorders in pregnant women and found an increase in LBPP associated with a high pre-gestational BMI (overweight/obesity) and weight gain over $15 \mathrm{~kg}$. The findings of Bliddal et al. [27] corroborate with our hypothesis to explain the absence of relation between weight gain and pain intensity.

\subsection{Clinical importance and study limitations}

This study is important clinically to incite health professionals to encourage pregnant women to adopt a healthy lifestyle, practicing physical activities regularly as a way to prevent low back and pelvic pain or the increase in its intensity. However, we stress that the physical activity should respect the specific characteristics of each pregnancy, as well as the muscular and cardiorespiratory training parameters.

This study's limitations were the lack of a specific questionnaire to evaluate the volunteers' level of physical activity and not including pregnant women in the first gestational trimester. Thus, we suggest new longitudinal studies to determinate the risk or protective factors related to the occurrence and intensity of LBPP in pregnant women.

\section{Conclusion}

Sedentary pregnant women have $30 \%$ more chances to have higher pain intensities when compared to the active women, regardless of the gestational trimester and weight gain.

\section{Conflict of interest}

None to report.

\section{References}

[1] Ehrlich GE. Low back pain. Bull World Health Organ. 2003; 81(9): 671-6.

[2] Gutke A, Betten C, Degerskär K, Pousette S, Fagevik Olsén $\mathrm{M}$. Treatments for pregnancy-related lumbopelvic pain: A systematic review of physiotherapy modalities. Acta Obstet Gynecol Scand. 2015; 94(11): 1156-67.

[3] Kendall KD, Emery CA, Wiley JP, Ferber R. The effect of the addition of hip strengthening exercises to a lumbopelvic exercise programme for the treatment of non-specific low back pain: A randomized controlled trial. J Sci Med Sport [Internet]. Sports Medicine Australia. 2015; 18(6): 626-31. Available from: http://dx.doi.org/10.1016/j.jsams.2014.11.006.

[4] Mota MJ, Cardoso M, Carvalho A, Marques A, Sa-Couto P, Demain S. Women's experiences of low back pain during pregnancy. J Back Musculoskelet Rehabil. 2015; 28(2): 3517.

[5] Mann L. Alterações biomecânicas durante o período gestacional: uma revisão. Motriz J Phys Educ. 2010; 16(3): 73041.

[6] Vleeming A, Albert HB, Östgaard HC, Sturesson B, Stuge B. European guidelines for the diagnosis and treatment of pelvic girdle pain. Eur Spine J. 2008; 17(6): 794-819.

[7] Ozdemir S, Bebis H, Ortabag T, Acikel C. Evaluation of the efficacy of an exercise program for pregnant women with low back and pelvic pain: A prospective randomized controlled trial. J Adv Nurs. 2015; 71(8): 1926-39.

[8] Mazicioglu M, Tucer B, Ozturk A, Serin IS, Koc H, Yurdakos K, et al. Low back pain prevalence in Turkish pregnant women. J Back Musculoskelet Rehabil [Internet]. 2006; 19: 89-96. Available from: http://www.deepdyve.com/lp/iospress/low-back-pain-prevalence-in-turkish-pregnant-womennsRX84Qkw3.

[9] Sd L, Pennick V. Interventions for preventing and treating low-back and pelvic pain during pregnancy (Review) Summary of Findings for the Main Comparison. 2015; (9).

[10] Lima AS, et al. Análise da postura e frequência de lombalgia em gestantes: estudo piloto. Journal of the Health Sciences Institute, Volta Redonda. 2011; 29(4): 290-293.

[11] Brito JLOP, et al. Lombalgia: prevalência e repercussões na qualidade de vida de gestantes. Revista de Enfermagem da UFSM, Santa Maria. 2014 abr/jun; 4(2): 254-264.

[12] Mann L, Kleinpaul JF, Teixeira CS, Konopka CK. Dor lombopélvica e exercício Físico durante a gestação. Fisioter. Mov. 2008 abr/jun; 21(2): 99-105.

[13] Moreira LS, de S Andrade SR, Soares V, De Avelar IS, Amaral WN, Vieira MF. Alterações posturais, de equilíbrio e dor lombar no período gestacional. Femina. 2011; 39(5): 241-4.

[14] OMS. Organização Mundial da Saúde, 1995. Physical Status: The use and interpretation of anthropometry. Technical Report Series, 854. Genebra: OMS.

[15] Atalah SE, Castillo CL, Castro RS. Propuesta de um nuevo estandar de evaluación nutricional em embarazadas. Rev Med Chile. 1997; 125: 1429-36.

[16] American College Obstetricians and Gynecologists, 2002.

[17] Da Silva SR, Sousa VPS, Bezerra DA, Ribeiro SO, Viana ESR. Influência da Cinesioterapia sobre a dor e qualidade de 
vida em mulheres grávidas. Fisioterapia Brasil. 2012; 13: 23 28.

[18] Evenson KR, et al. Guidelines for physical activity dur ing pregnancy: comparisons from around the world. Am. J. Lifestyle Med. 2014; 8(2): 102-121.

[19] Kokic IS, et al. Effect of therapeutic exercises on pregnancyrelated low back pain and pelvic girdle pain: secondary analysis of a randomized controlled trial. J Rehabil Med. 2017; 49: 251-257.

[20] Gallo-Padillaa D, Gallo-Padilla C, Gallo-Vallejoc FJ, GalloVallejo JL. Lumbalgia durante el embarazo. Abordaje multidisciplinar. Semergen. 2016; 42(6): e59-e64.

[21] Sabino J, Grauer JN. Pregnancy and low back pain. Curr Rev Musculoskelet Med. 2008; 1(2): 137-141.

[22] De Sousa VPS, Ribeiro SO, Aquino CMR, Viana ESR. Quality of sleep in pregnant woman with low back pain. Fiosterapia em Movimento (on-line). 2015; 28: 319-326.

[23] Garland M. Physical activity during pregnancy: a prescription for improved perinatal outcomes. The Journal for Nurse Practitioners - JNP. January 2017; 13(1).
[24] Fazzi C, Saunders DH, Linton K, Norman JE, Reynolds RM. Sedentary behaviours during pregnancy: a systematic review. International Journal of Behavioral Nutrition and Physical Activity. 2017; 14: 32.

[25] Leitão MB, et al. Posicionamento oficial da sociedade brasileira de medicina do esporte: atividade física e saúde na mulher. Rev Bras Med Esporte. 2000; 6: $\mathrm{N}^{\circ} 6$ - Nov/Dez.

[26] Ghaderi F, Jafarabadi MA, Bandpei MAM. Prevalence of musculoskeletal pain and associated factors with low back pain during pregnancy. Iranian Journal of Obstetrics, Gynecology and Infertility. 2013; 15(41): 9-16.

[27] Bliddal M, et al. Association of pre-pregnancy body mass index, pregnancy-related weight changes, and parity with the risk of developing degenerative musculoskeletal conditions. Arthritis and Rheumatology. 2016; 68(5): 1156-1164. 